\title{
An Efficient algrithm to find All 'Bidirectional' Edges of an Undirected Graph.
}

\author{
B. Mishra,
}

\section{Department of Computer Science, Carnegie-Mellon University Pittsburgh, PA 15213}

\begin{abstract}
ABSTRAC'T. An efficient algorithm for the All-Bidirectional-Filges Problem is presented. The All-BidirectionalEdges Problem is to find an edge-labelling of an undirected network, $G=\left(V, E^{\prime}\right)$, with a source and a sink, such that an edge $[u, v] \in E^{\prime}$ is labelled $\langle u, v\rangle$ or $\langle v, u\rangle$ (or both) depending on the existence of a (simple) path from the source to sink that visits the vertices $u$ and $v$, in the order $u, v$ or $v, u$, respectively. The algorithm presented works by partitioning the graph into a set of bridges and analyzing them recursively. The time complexity of the algorithm is shown to be $O(|E| \cdot|V|)$.

The problem arises naturally in the context of the simulation of an MOS transistor network, in which a transistor may oprrate as a unilateral or a bilateral device, depending on the voltages at its source and drain nodes. For efficient simulation, it is required to detect the set of transistors that mily operate as bilateral devices. Also, this algorithm can be used in order to detect all the sneak paths in a network of pass transistor.
\end{abstract}

\section{Introduction}

Let $G=(V, E)$ be a finite undirected graph with two distinguished vertices, the source, $s$, and the sink, $t$. We call an edge $e=[u, v]$ of $G$ 'bidirectional', if there are two simple paths connecting $s$ and $t$ and traversing $e$ in either order $-u, v$ and $v, u$. Similarly, we call an edge $e=[u, v]$ of $G$ 'unidirectional', if every simple path connecting $s$ and $t$ and containing $e$, traverses $e$ only in one order, say $u$, $v$; additionally, $e$ is labelled $\langle u, v\rangle$. The All-BidirectionalEdges problem is to find all the 'bidirectional' and 'unidirectional' edges of $G$, together with the labellings of the 'unidirectional' edges.

From an alternative formulation of the problem it is easy to see that we can label each edge $[u, v] \in E$ by asking two questions: (i) Are there two disjoint paths $s \stackrel{* *}{\longrightarrow} u$ and $v \stackrel{*}{\longrightarrow} t$ ? (ii) Are there two disjoint paths $s \stackrel{*}{\longrightarrow} v$ and $u \stackrel{*}{\rightarrow} t$ ? There are $O(|E| \cdot|V|)$ time algorithms to find two vertex disjoint paths in an undirected graph, independently discovered by Ohtsuki(1980)[7], Seymour(1980)[9] and Shiloach(1980) [10]. The naive way of solving the All-Bidirectional-Edges problem is to invoke an algorithm

This work was supported in part by NSF grant MCS-8216706.

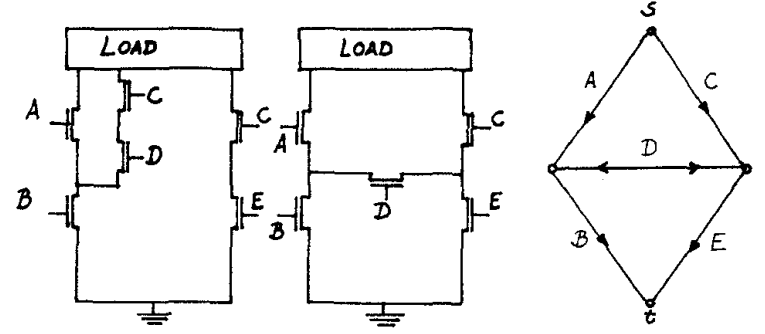

Figure 1: $(a))$ A circuit for $\neg((A \wedge B) \vee(B \wedge C \wedge D) \vee(C$ $\wedge E))$. (b)) The resulting subgraph after common subcircuit climination. However, because of the sneak path through $D$, it is, in fact, a circuit for $\neg((A \wedge B) \vee(B \wedge C \wedge D) \vee(C \wedge E)$ $\vee(A \wedge D \wedge E))$. (c)) The corresponding graph in which $D$ is 'bidirectional' and all other edges are 'unidirectional.'

for Two-Disjoint-Path twice per each edge. This takes $O\left(|E|^{2} \cdot|V|\right)$. On the other hand, existence of an algorithm for All-Bidirectional-Edges problem, with a timecomplexity lower than $O(|E| \cdot|V|)$, readily, results in a similarly efficient algorithm for Two-Disjoint-Paths problem, which is unlikely.

In this paper, we describe an $O(|E| \cdot|V|)$ time algorithm for All-Bidirectional-Edges problem, using a completely new approach. The algorithm makes a novel use of bridges of a circuit in a general graph.

The problem of finding all 'bidirectional' edges arises naturally in the context of the simulation of an MOS transistor network, in which a transistor may operate as a unilateral or a bilateral device, depending on the voltages at its source and drain nodes. (Cf. Brand (1983)[2], Frank (1984)[5].) For efficient simulation, it is important to find the set of transistors that may operate as bilateral devices. Also, sometimes it is desired that information propagates in one direction only, and propagation in the wrong direction (resulting in a sneak path) can cause functioral error. (See Figure 1) Cur algorithm can be used to detect all the sneak paths.

\section{Preliminaries}

This section introduces some useful graph theoretic terms. The term 'bridge' is taken from Tutte(1977)[13]. 


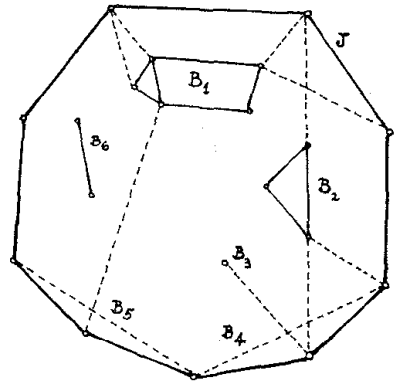

Figure 2: Bridges of $J$. Bridges $B_{1}, B_{2}, B_{3}$ and $B_{6}$ are proper bridges, and $B_{4}$ and $B_{5}$ are degenerate bridges. The bridges $B_{1}$ and $B_{2}$ interlace.

Definition 1.1: A vertex of attachment of a subgraph $H$ of $G$ is a vertex of $H$ that is incident in $G$ with some edge not belonging to $H$. Let $J$ be any circuit of $G$. We define a bridge of $J$ in $G$ as any subgraph $B$ that satisfies the following three conditions:

1. Each vertex of attachment of $B$ is a vertex of $J$.

2. $B$ is not a subgraph of $J$.

3. No proper subgraph of $B$ satisfies the above conditions.

Definition 1.2: (Cf. Figure 2.) An edge $e=[u, v]$ of $G$ not belonging to $J$ but having both ends in $J$ is referred to as a degenerate bridge. Let $G$ be the graph derived from $G$ by deleting the vertices of $J$ and all their incident edges. Let $C$ be any component of $G^{-}$Let $B$ be the subgraph of $G$ obtained from $C$ by adjoining to it each edge of $G$ having one end in $C$ and one in $J$, and adjoining also the ends in $J$ of all such edges. The subgraph $B$ satisfies the conditions to be a bridge. Such a bridge is called proper. The component $C$ of $G^{-}$is the nucleus of $B$.

Definition 1.3: Let the vertices of attachment of a bridge $B$ of $J$ be $W(G, B)=\left\{v_{0}, v_{1}, \ldots, v_{k-1}\right\}$, where $v_{0}, v_{1}, \ldots$, $v_{k-1}$ is their enumeration in the cyclic order on $J$. The vertices of attachment dissect $J$ into $k$ subpaths $L_{0}, L_{1}$, $\ldots, L_{k-1}$ such that $L_{j}=J \llbracket v_{j} ; v_{j+1}(\bmod k) \rrbracket$. These subpaths are called the residual paths of $B$ in $J . w(G, B)$ will stand for $|W(G, B)|$.

Definition 1.4: Let $B_{1}$ and $B_{2}$ be two distinct bridges of a circuit $J$ of $G$.

- We say $B_{1}$ avoids $B_{2}$ if and only if one of the following two conditions is satisfied:

1. $w\left(G, B_{1}\right) \leq 1$ or $w\left(G, B_{2}\right) \leq 1$.

2. All the vertices of attachment of $B_{1}$ are contained in a single residual path $L$ of $B_{2}$.

- If $B_{1}$ and $B_{2}$ do not avoid one another we say that they overlap.

- If there exist two vertices of attachment $x_{1}$ and $x_{2}$ of $B_{1}$ and two vertices of attachment $y_{1}$ and $y_{2}$ of $B_{2}$, all four distinct, such that $x_{1}$ and $x_{2}$ separate $y_{1}$ and $y_{2}$ in the circuit $J$, then we say that they interlace.

- If $B_{1}$ and $B_{2}$ have exactly the same set of vertices of attachment we say that they are equivalent.

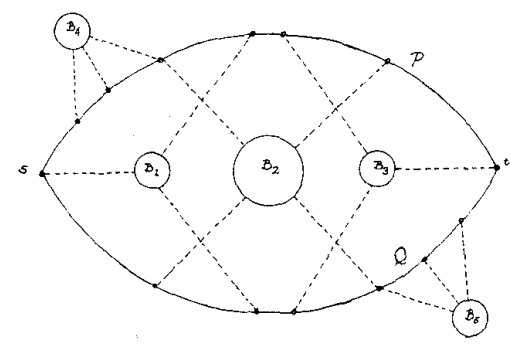

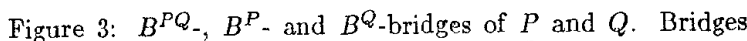
$B_{1}, B_{2}$ and $B_{3}$ are $B^{P Q}$-bridges; $B_{4}$ is a $B^{P}$-bridge and $B_{5}$, a $B^{Q}$-bridge.

Definition 1.5: Let $J$ be a circuit of the graph $G$. A path $N$ in $G$ avoiding $J$ but having its two ends $x$ and $y$ in $J$ is called a cross-cut of $J$ from $x$ to $y$.

Definition 1.6: (Cf. Figure 3.) Let $J$ be a circuit of the graph, $G$ such that $s$ and $t$ lie on $J$. Let the subpath $J \llbracket s ; t \rrbracket$ be $P$ and its complomentary subpath in $J$ be $Q$. The bridges of $J$ are classified as follows:

- $B^{P Q}$-BrIDGES: The set of bridges with at least one vertex of attachment on $P \rrbracket s ; t \llbracket$ and at least one vertex of attachment on $Q \rrbracket s ; t \llbracket$.

- $B^{P}$ - BRIDGES: The set of bridges with at least one vertex of attachument on $P \rrbracket s ; t \llbracket$ and no vertex of attachment on $Q \rrbracket s ; t \|$.

- $B^{Q}$-BRIDGES: The set of bridges with no vertex of attachment on $P \rrbracket s ; t[$ and at least one vertex of attachment on $Q \rrbracket s ; t \llbracket$.

Definition 1.7: Let $J, P$ and $Q$ be as in the previous definition. Then $J$ is called an ambitus if every $B^{P}$ - or $B^{Q}$-bridge avoids every $B^{P Q}$-bridge.

Definition 1.8: Let $J, P$ and $Q$ be as before and $B=$ $B_{1}, \ldots, B_{k}$ be the $B^{P}$-bridges with respect to $P$. A nonempty subset of bridges $B_{n} \subseteq B$ is called a block of $B^{P}$. bridges if it satisfies the following two conditions:

1. If $B_{i} \in \mathbf{B}_{n}$ and $B_{i}$ and $B_{j}$ overlap, then $B_{j} \in \mathbf{B}_{n}$.

2. No non-empty proper subset of $\mathbf{B}_{n}$ satisfies the preceding condition.

Definition 1.9: Let $G=(V, E)$ be a graph. The tree $T=\left(V^{\prime}, E^{\prime}\right)$ of the graph $G$ is an undirected graph such that: The set of vertices $V^{\prime}$ correspond with the nonseparable components (green) and the separation vertices (blue); and the edges of $T$ connect green vertices to blue vertices if and only if the nonseparable component contains the separation vertex.

Definition 1.10: A graph $G=(\dot{V}, E)$ with distinguished vertices $s$ and $t,(G ; s, t)$ is said to be a chain graph, if the tree of the graph $G$ is a path $P$ from $C_{s}$ to $C_{t}$ consisting of alternating green and blue vertices, where $C_{s}$ and $C_{t}$ are the nonseparable components of $G$ containing the vertices $s$ and $t$, respectively.

We present the following propositions that will be used quite often later on. 
Proposition 1.11: (Even[3]) Let $x, y$ and $z$ be three distinct vertices of attachment of a bridge. $B$ of $J$ in $G$. Then there is a vertex $v$ belonging to the nucleus of $B$ for which there are three internally vertex disjoint paths in $B: Y_{1} \llbracket x ; v \rrbracket, Y_{2} \| ; ; v \rrbracket$ and $Y_{3} \| z ; v \rrbracket$.

Following Tutte[14], we define a $Y$-graph as the union $Y$ of three paths $Y_{1}, Y_{2}$ and $Y_{3}$ which have one end $v$ in common but are otherwise mutually disjoint. We call $v$ the center and the paths $Y_{i}$ the arms of $Y$.

Proposition 1.12: Let $x, y$ and $z$ be three distinct vertices of attachment of bridge $B$ of $J$ in $G$ and let $e$ be an edge of $B$ such that there is a cross-cut of $J$ between $x$ and $y$ containing $e$. Then at least one of the two following conditions is satisfied: (1) there is a cross-cut of $J$ between $x$ and $z$ containing $e$ (2) there is a cross-cut of $J$ between $y$ and $z$ containing $e$.

PROOF. The proof is similar to that of the proposition 1.11 and hence, omitted.

Proposition 1.13: (Tutte[13]) Let $B_{1}$ and $B_{2}$ be distinct overlapping bridges of a circuit $J$ of $G$. Then either $B_{1}$ and $B_{2}$ interlace or they are equivalent 3-bridges.

\section{Overview}

For the ease of exposition, the algorithm is presented in two parts: The first part is simple but provides an algorithm of time-complexity of $O\left(|E| \cdot|V|^{2}\right.$ ) (to be improved later); it also identifies a graph of suitable structure (called a TYPE.IV graph) such that an efficient algorithm for such graph yields an efficient algorithm for general graphs. The second part, on the other hand, is more complicated but provides an $O(|E| \cdot|V|)$ time algorithm for a TYPE.IV graph, which, in turn, implies an $O(|E| \cdot|V|)$ time algorithm for the general graphs.

In the first part (Section 3), we introduce four different classes of graphs: TYPE.I, TYPE.II, TYPE.III and TYPE.IV graphs, of successively simpler structure and show that we can label the graphs of a particular type efficiently, on the assumption of existence of efficient algorithms for the subsequent types. In the process we give a set of four mutually ${ }^{\dagger}$ recursive algorithms such that these, together with an algorithm for TYPE.IV graph of time complexity $O(T(|E|,|V|)) \geq O(|E| \cdot|V|)$, will result in an $O(T(|E|,|V| j)$ algorithm for general graphs. The first part concludes with an $O\left(|E| \cdot|V|^{2}\right)$ algorithm for TYPE.IV graphs.

In the second part (Section 4) we sketch an $O(|E| \cdot|V|)$ time algorithm for TYPE.IV graph. This is a Divideand-Conquer algorithm and relies on an important concept, called a U-Frcgment. Intuitively, a U-Fragment can be thought of as a super node which can be entered or exited through its end vertices $\left(s^{\prime}\right.$ and $\left.t^{\prime}\right)$ or its upper and lower external attachments. The task is to find suit-

t. Analysis of a TYPE.III graph may result in a call to the main algorithm. able vertex disjoint paths in this super node, connecting end vertices and external attachments. These paths can be extended to appropriate vertex-disjoint paths in the outer U-Fragment (recursively) or in a TYPE.IV graph. Such paths are guaranteed to exist if and only if the UFragment is feasible (Definition 4.2) and has certain disjoint pairs of Cross-Cuts. The main idea of the algorithms is to find such paths and to use them to label the edges.

\section{A Simple Algorithm}

\subsection{Assumptions and Classification}

Assumption 3.1: The graph, $G$, is a finite connected undirected strict graph.

Assumption 3.2: The graph, $G$, is a chain graph.

Justification for the Assumption 3.2. Let $P$ be a path in the tree of $G, T(G)$ from $C_{s}$ to $C_{t}$ consisting of alternating green and blue vertices. Let $C_{s}=C_{1}, C_{2}, \ldots, C_{m}=C_{t}$ be the nonseparable components (green) and $a_{1}, a_{2}, \ldots, a_{m-1}$ the separation vertices (blue) on $P$. Let $s_{1}=s, t_{m}=t$ and $s_{i}=a_{i-1}(1<i \leq m)$ and $t_{i}=a_{i}(1 \leq i<m)$. Let $E_{1} \subseteq E$ be the edges of the $C_{i}$ 's and $E_{2}=E \backslash E_{1}$.

Theorem 3.3: Let $G=(V, E)$ be an undirected network with a source $s$ and sink $t$ and let $E_{1}$ and $E_{2}$ be the partition of the edges, $E$ as described. For each edge $e \in E$, $e \in E_{2}$ iff there is no simple path from $s$ to $t$ containing e.

In a pre-processing step, we find and delete the edges, $E_{2}$ to obtain the graph $G^{\prime}$, and present each non-separable component $\left(C_{i} ; s_{i}, t_{i}\right)$ of $G^{t}$ as input to the main algorithm. The preprocessing step takes $O(|E|+|V|)$ time.

Definition 3.4: We introduce a classification of graphs as follows: (i)A nonseparable graph is said to be of type $I$, if it has a circuit $J$ containing the vertices $s$ and $t$ and all its bridges are $B^{P_{-}}, B^{Q_{-}}$or $B^{P Q_{-}}$-bridges; (ii) type $I I$, if it is of TYPE.I and all its bridges are of $B^{P Q_{-}}$bridges; (iii) type $I I I$, if it is of TYPE.II and has only a single $B^{P Q}$-bridge; and (iv) type $I V$, if it is of TYPE.III and the subgraph, derived by deleting the vertices $s$ and $t$ together with their incident edges, is nonseparable.

\subsection{Labelling a Chain Graph}

Assume that we have an algorithm, called L,ABELTYPE-I to label the edges of a TYPE.I graph.

\section{Algorithm LABEL-GRAPH. (Cf. Figure 4.)}

-STEP1. Find the nonseparable components of the graph. Let $C_{1}, C_{2}, \ldots, C_{m}$ be the chain of nonseparable components and let $s_{i}$ and $t_{i}$ be the vertices associated with $C_{i}$ (Cf. Assumption 3.2). For each $\left(C_{i} ; s_{i}, t_{i}\right)$, where $1 \leq i \leq m$, do the following steps.

-STEP2. Find a circuit $J$ containing the vertices $s_{i}$ and $t_{i}$ in $C_{i}$. Since $C_{i}$ is nonseparable such a circuit exists and can be found in $O(|E|)$ time, using depth-first search 

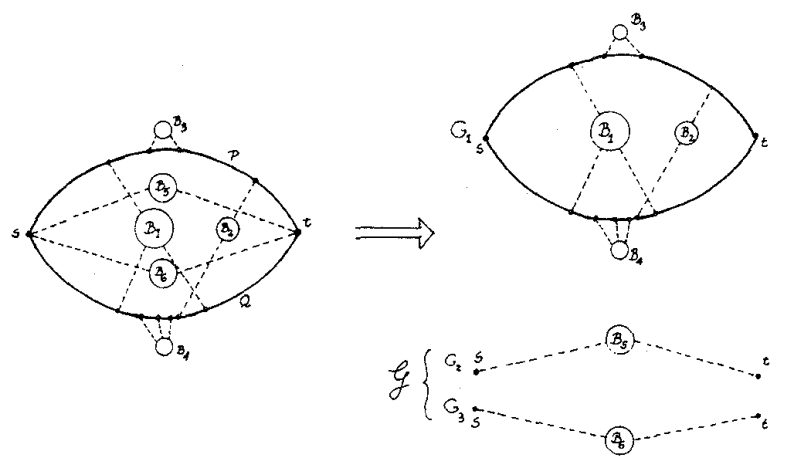

Figure 4: Steps of the Algorithm LABEL-GRAPH.

technique. We call the subpath $J \llbracket s_{i} ; t_{i} \rrbracket, P$; and its complementary residual path in $J, Q$.

- STEP3. Find all the bridges of $C_{i}$ with respect to the circuit $J$. Let $\mathcal{G}=\left\{G_{2}, G_{3}, \ldots, G_{k}\right\}$ be the set of bridges with only attachments $s_{i}$ and $t_{i}$; and let $B$ be the set of $B^{P}$. , $B^{Q}$ - and $B^{P Q_{-}}$bridges of $J$. Let $G_{1}$ be the graph derived from $C_{i}$ by deleting the bridges of $\mathcal{G}$. Since $G_{1}$ is a TYPE. I graph, by assumption we can label the edges of $G_{1}$ using the algorithm LABEL-TYPE-I. Moreover, since $G_{i} \in \mathcal{G}$ is a chain graph, the labelling for $G_{i}$ can be done by recursively calling the main algorithm. We can find the bridges in $O(|E|)$ time as in the planarity testing algorithm.

Theorem 3.5: Assume that LABEL-TYPE-I correctly labels the edges of a TYPE.I graph. Then the algorithm LABEL-GRAPH correctly labels the edges of a nonseparable graph.

\subsection{Labelling a Type. I Graph}

Assume that we have an algorithm, called LABELTYPE-II, to label the edges of a TYPE.II graph.

Algorithm LABEL-TYPE-I.(Cf. Figure 5.)

-STEP 1. Modify $J$ to obtain a circuit $J^{\prime}$ such that $J^{\prime}$ is an ambitus. Let $\mathbf{B}_{1}, \mathbf{B}_{2}, \ldots, \mathbf{B}_{n}$ be its blocks of $B^{P}$. and $B^{Q}$-bridges; and $s_{i}$ and $t_{i}$, the left-and the right-most

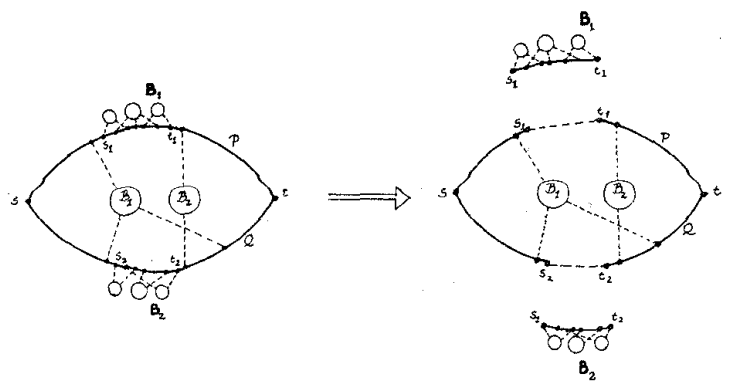

Figure 5: Steps of the Algorithm LABEL-TYPE-I. vertices of attachments of the bridges of $\mathbf{B}_{i}$, respectively. Let $G^{\prime \prime}$ be the graph derived from $G$ by first deleting all the blocks $\mathbf{B}_{i}$ and then replacing $P \llbracket s_{i} ; t_{i} \rrbracket$ or $Q \llbracket s_{i} ; t_{i} \rrbracket$. (depending on whether $\mathrm{B}_{i}$ is a block of $B^{P}$. or $B^{Q_{-} \text {bridges, }}$ respectively) by a single link $\left[s_{i}, t_{i}\right]$. we refer to $\left[s_{i}, t_{i}\right]$ as a pseudo-edge (l) of the block $\mathrm{B}_{i}$. This step can be done in time $O(|E|+|V|)$. A linear time algorithm for finding the ambitus can be found in the full paper.

-STEP2. Since graph $G^{\prime \prime}$ is a TYPE.II graph, by assumption, we can label the edges of $G^{\prime \prime}$ using the algorithm LABEL-TYPE-II.

-STEP 3. For each block $\mathrm{B}_{i}$, where $1 \leq i \leq n$, do the following: If tihe pseudo-edge of $\mathbf{B}_{i}$ is labeled 'bidirectional' by the Step. 2 , then all edges of $\mathbf{B}_{i}$ are 'bidirectional'; otherwise, label the edges of $\mathbf{B}_{i}$ by calling LABEL-GRAPH with the argument $\left(\mathbf{B}_{i} ; s_{i}, t_{i}\right)$.

Let $G$ be a TYPE.I graph with circuit $J=P \cup Q$. Let $\mathbf{B}_{i}$ be a block of $B^{P}$-bridges of $G$ and let its left- and right-most vertices of attachment be $s_{i}$ and $t_{i}$.

Lemma 3.6: Let $G^{\prime}$ be the graph derived by first deleting $\mathbf{B}_{i}$ from $G$ and then replacing the subpath $P \llbracket s_{i} ; t_{i} \rrbracket$ by $a$ single link $\left[s_{i}, t_{i}\right]$, called the pseudo-edge of $\mathbf{B}_{i}$. (i) If in a legal labelling of $G^{\prime},\left[s_{i}, t_{i}\right]$ is labelled 'bidirectional' then all edges of $\mathbf{B}_{i}$ are 'bidirectional'. (ii) If in a legal labelling of $G^{\prime},\left[s_{i}, t_{i}\right]$ is labelled $\left\langle s_{i}, t_{i}\right\rangle$ then the labelling of the edges of $\mathbf{B}_{i}$ are determined by that of $\left(\mathbf{B}_{i} ; s_{i}, t_{i}\right)$.

Lemma 3.7: Let $G^{\prime \prime}$ be the graph derived from $G$ by re-

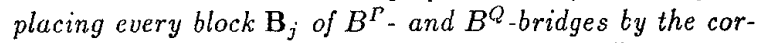
responding pseudo-edge $\left[s_{j}, t_{j}\right]$. Then $\ell\left(\left[s_{i}, t_{i}\right]\right)$ in $G^{\prime}=$ $\ell\left(\left[s_{i}, t_{i}\right]\right)$ in $G^{\prime \prime}$.

Theorem 3.8: Suppose LABEL-TYPE-II correctly labels the edges of a TYPE. I graph. Then the algorithm LABELTYPE-I correctly labels the edges of a TYPE.I graph.

PROOF. Follows from the previous two Lcmmas.

\section{4. 'Labelling a Type.II Graph}

We assume that we have an algorithm, called LABELTYPE-III to label the edges of a TYPE.III graph.

Algorithm LABEL-TYPE-II. (Cf. Figure 6.)

-STEP 1. Let $B_{1}, B_{2}, \ldots, B_{n}$ be $B^{P O}$-bridges of $J$ in $G$. We modify each bridge fragment $G_{i}=B_{i} \cup J$ as follows: Let $s_{P}$ and $t_{P}$ be the left- and right-most vertices of attachment of $B_{i}$ on $P$; and, similarly, $s_{Q}$ and $t_{Q}$, on $Q$. Let $L_{0}, L_{1}, \ldots, L_{k}$ be its residual paths not containing $s$ or t. $G_{i}^{\prime}$ is derived from $G_{i}$ by contracting the subpaths $P \llbracket s ; s_{P} \rrbracket, Q \llbracket\left[s ; s_{Q} \rrbracket, P \llbracket t_{P} ; t \rrbracket, Q \llbracket t_{Q} ; t \rrbracket, L_{0}, L_{1}, \ldots, L_{i}\right.$ to single links, called a pseudo-edge(II) of the subpath. $J^{\prime}$ is the circuit in $G_{i}^{\prime}$ derived from $J$ by the contraction. Since, each such $G_{i}^{\prime}$ is a TYPE.III graph, by assumption, we can label the edges of $G_{i}^{\prime}$ using the algorithm TYPE.III.

-STEP 2. For each edge $e$ of $J$, let $\left\{e_{1}^{\prime}, e_{2}^{\prime}, \ldots, e_{n}^{\prime}\right\}$ be the set of edges of $G_{i}^{\prime}$ such that $e_{i}^{\prime}$ is the contraction of a subpath containinge. Then if any $e_{i}^{\prime}$ is 'bidirectional' mark $e$ 'bidirectional'. This step can be done in time $O(|E|)$, 


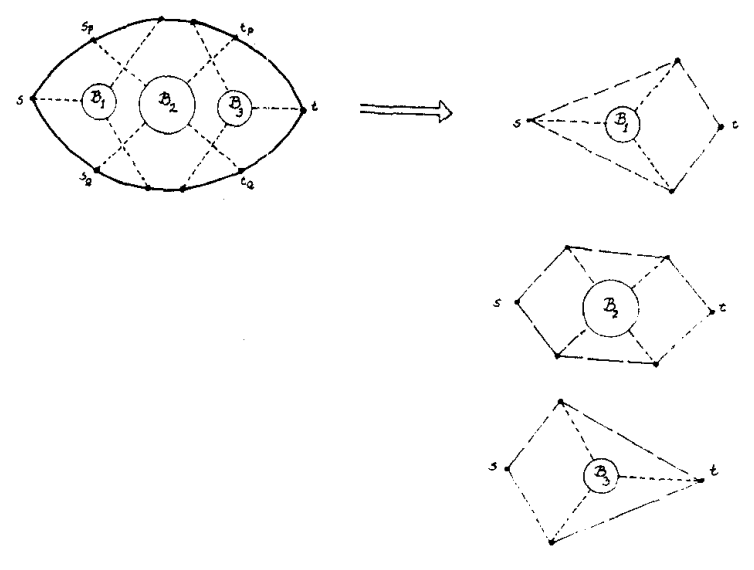

Figure 6: Steps of the Algorithm LABEL-TYPE-II.

since, as it will be shown in Lemma 3.14 there are at most $4 \cdot|E|$ pseudo-edges(II).

-STEP 3. Let $B_{i}$ and $B_{j}$ be two interlacing $B^{P Q_{-} \text {bridges of }}$ $J$ in $G$ and $s_{P}$ and $t_{P}$ (respectively, $s_{Q}$ and $t_{Q}$ ), their leftand right-most vertices of attachment on $P$ (respectively, $Q)$, distinct from $s$ and $t$. Label the edges of the subpaths $P \llbracket s_{P} ; t_{P} \rrbracket$ and $Q \llbracket s_{Q} ; t_{Q} \rrbracket$ 'bidirectional'. This step can be done in time $O(|E|)$.

Lemma 3.9: Let $E$ be a $B^{P Q}$-bridge of $J=P \cup Q$ in TYPE.II graph $G$ and $e=[u, v]$, an edge of $B$. Let $G^{\prime}$ be a subgraph of $G$ derived from $G$ by deleting all bridges except $B$ and contracting the residual suhpaths as in the Algorithm LABEL-TYPE-II. Then there is a path from $s$ to $t$ in $G$ traversing $e$ in the order $u, v$ if and only if there is a path from s to $t$ in $G^{\prime}$ traversing $e$ in the same order.

PROOF. $(\Leftrightarrow)$ Trivially true, since $G^{\prime}$ is a minor of $G .(\Rightarrow)$ If $R \llbracket s ; t \rrbracket$ traverse $e$ in the order $u$ and $v$ then we can write $R \llbracket s ; t \rrbracket$ as $R \llbracket s ; x \rrbracket * R\|x ; y \rrbracket * R\| y ; t \rrbracket$ such that $R \llbracket x ; y \rrbracket$ is a cross-cut of $J$, belonging to $B$ and traversing $e$ in the order $u, v$. If $x \in P \llbracket s ; t \rrbracket$ and $y \in Q \llbracket s ; t \rrbracket$ then the path $P \llbracket s ; x \rrbracket * R \llbracket x ; y \rrbracket * Q \llbracket y ; t \rrbracket$ in $G^{\prime}$ traverses $e$ in the same order. Hence, assume that $x, y \in P \rrbracket s ; t$. Since $B$ is $B^{P Q}$-bridge it has a vertex of attachment $z \in Q \rrbracket s ; t$. Using proposition 1.12, we can find simple paths from $s$ to $t$ such that they traverse $e$ in either direction.

Lemma 3.10: Let $B_{i}$ and $B_{j}$ be two interlacing $B^{P Q}$. bridges of $J=P \cup Q$. Let $s_{P}$ and $t_{P}$ be the left-and right-most vertices of attachment of $B_{i}$ and $B_{j}$ on $P$ distinct from $s$ and $t$; and similarly $s_{Q}$ and $t_{Q}$ on $Q$. Then the edges of the residual paths $P \llbracket s_{P} ; t_{P} \rrbracket$ and $Q \llbracket s_{Q} ; t_{Q} \rrbracket$ are 'bidirectional'.

PROOF. (Cf. Figure 7.) Since $B_{i}$ and $B_{j}$ interlace, there exist two vertices of attachment $a_{i}$ and $b_{i}$ of $B_{i}$ and two vertices of attachment $a_{j}$ and $b_{j}$ of $B_{j}$, all four distinct, such that $a_{i}$ and $b_{i}$ separate $a_{j}$ and $b_{j}$ in the circuit $J$. Let $N_{i}$ be a cross-cut of $J$
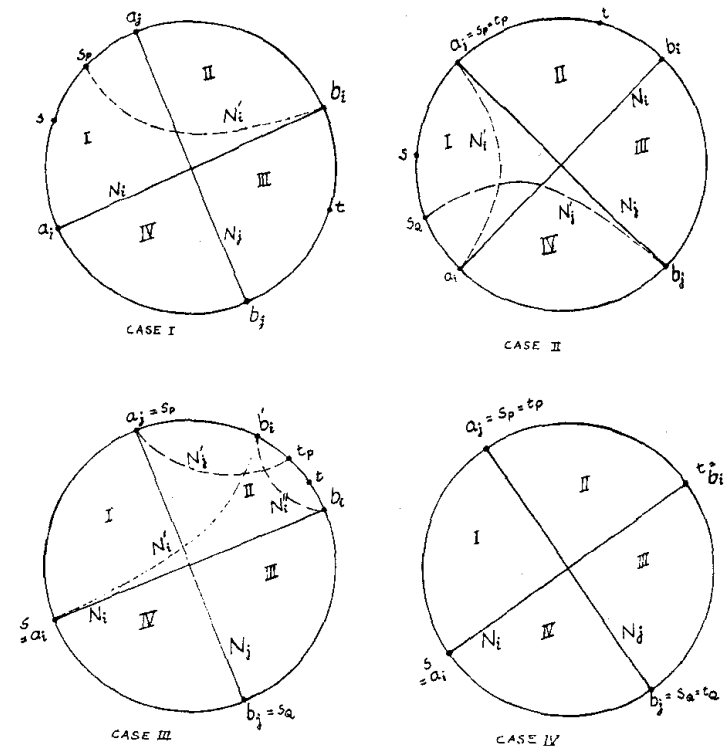

Figure 7: Four Cases of the Lemma.3.10

in $G$ between $a_{i}$ and $b_{i}$ belonging to $B_{i}$; and $N_{j}$ between $a_{j}$ and $b_{j}$ belonging to $B_{j}$. The four residual paths $J \llbracket a_{i} ; a_{j} \rrbracket, J \llbracket a_{j} ; b_{i} \rrbracket$, $J \llbracket b_{i} ; b_{j} \rrbracket$ and $J \llbracket b_{j} ; a_{i} \rrbracket$ will be referred to as quadrants I, II, III and IV, respectively. The distinguished vertices $s$ and $t$ lie on $J$. Observe that if two $B^{P Q}$-bridges $B_{i}$ and $B_{j}$ interlace then it is always possible to choose four distinct vertices $a_{i}, b_{i}, a_{j}$ and $b_{j}$ such that $s$ and $t$ do not lie in the same quadrant.

Hence there are four cases to consider:

-CASE. $1 s$ is an internal vertex of quadrant $I$ and $t$ is an internal vertex of quadrant III. Let $J \llbracket s ; t \rrbracket$ in quadrants I, II and III be $P \| s ; t \rrbracket$ and $Q \llbracket s ; t \rrbracket$ its complement in $J$. Let $a_{i}, b_{i}, a_{j}$ and $b_{j}$ be modified such that $a_{i}$ and $a_{j}$ are the left-most vertices of attachment of $B_{i}$ and $B_{j}$, distinct from $s$, on $Q$ and $P$ respectively; and similarly, $b_{i}$ and $b_{j}$, distinct from $t$. Accordingly, $N_{i}$ and $N_{j}$ are modified. Observe that $s_{P}$ (respectively, $s_{Q}$ ) is distinct from $a_{j}$ (respectively, $\dot{a}_{i}$ ) if and only if $s_{P}$ is the left-most vertex of attachment $B_{i}$ on $P$ (respectively, $s_{Q}$, the left-most vertex of attachment $B_{j}$ on $Q$ ), distinct from $s$. A similar observation can be made about $t_{P}$ and $t_{Q}$.

Every edge of the residual paths $P \llbracket a_{j} ; b_{i} \rrbracket$ and $Q \llbracket a_{i} ; b_{j} \rrbracket$ is 'bidirectional'. The paths $P \llbracket s ; t \rrbracket$ and $Q \llbracket s ; a_{i} \rrbracket * N_{i} \llbracket a_{i} ; b_{i} \rrbracket * P \llbracket b_{i} ; a_{j} \rrbracket *$ $N_{j} \llbracket a_{j} ; b_{j} \rrbracket * Q \llbracket b_{j} ; t \rrbracket$ traverse the edges of $P \llbracket a_{j} ; b_{i} \rrbracket$ in either direction. Similarly, for $Q \llbracket a_{i} ; b_{j} \rrbracket$.

If $s_{p}$ and $a_{j}$ are distinct then every edge of the path $P \llbracket s_{P} ; a_{j} \rrbracket$ is 'bidirectional'. Since $s_{P}$ is a vertex of attachment of $B_{i}$, there is a cross-cut between $s_{P}$ and $b_{i}$, say $N_{i}^{\prime}$. then the paths $P \llbracket s ; t \rrbracket$ and $\left.Q \llbracket s ; b_{j} \rrbracket * N \llbracket b_{j} ; a_{j} \rrbracket * P \llbracket a_{j} ; s_{P} \llbracket * N_{i} \llbracket s_{P} ; b_{i} \rrbracket * P \llbracket b_{i} ; t\right]$ traverse the edges of $P \llbracket s_{p} ; a_{j} \rrbracket$ in either directions. Similarly, for $P \llbracket b_{i} ; t_{\Gamma} \rrbracket, Q \llbracket s_{Q} ; a_{i} \rrbracket$ and $Q \llbracket b_{j} ; t_{Q} \rrbracket$.

-CASE. $2 s$ is an internal vertex of quadrant $I$ and $t$, of quadrant $I I$. Let $J \llbracket s ; t \rrbracket$ in quadrants I and II bc $P \llbracket s ; t \rrbracket$ and $Q \llbracket s ; t \rrbracket$ its complement in $J$. Wo may assume that $a_{j}=s_{P}=t_{P}$. Since, otherwise, we can reduce this case to an instance of the case(1).

Since $B_{i}$ is a $B^{P Q_{\text {-bridge, }}} a_{j}$ is also an attachment of $B_{i}$; and there are cross-cuts between $a_{j}$ and $a_{i}\left(N_{i}^{\prime}\right)$ and betwcen $a_{j}$ 
and $b_{i}\left(N_{i}^{\prime \prime}\right)$. Let $a_{i}$ and $b_{i}$ be modified to be the left- and right-most vertices of attachment of $B_{i}$ on $Q$, distinct from $s$ and $t$, respectively. Accordingly $N_{i}$ is noodified. Observe that $s_{Q}$ is distinct from $a_{i}$ if and only if $s_{Q}$ is the left-most vertex of attachment of $B_{j}$ on $Q$ distinct from s. A similar relation holds between is and $b_{i}$.

Every edge of the residual path $Q \llbracket a_{i} ; b_{j} \rrbracket$ and $Q \llbracket b_{j} ; b_{i} \rrbracket$ is 'bidirectional'. The paths $Q \llbracket s ; t \rrbracket$ and $P \llbracket\left[s ; a_{j} \rrbracket * N_{j} \llbracket a_{j} ; b_{j} \rrbracket * Q \llbracket b_{j} ; a_{i} \rrbracket *\right.$ $\left.N_{i} \rrbracket a_{i} ; b_{i} \rrbracket * Q \llbracket b_{i} ; t\right]$ traverse edges of $Q \llbracket a_{i} ; b_{j} \rrbracket$ in eithor directions. Similarly, for $Q \llbracket b_{j} ; b_{i} \rrbracket$.

If $s_{Q}$ and $a_{i}$ are distinct, then every edge of $\left.Q \llbracket s_{Q} ; a_{i}\right]$ is 'bidirectional'. Since $s_{Q}$ is a vertex of attachment of $B_{j}$, there is a crosscut between $s_{Q}$ and $b_{j}$, saty $N_{j}^{\prime}$. The paths $Q[s ; t]$ and $P\left[s ; a_{j}\right] *$ $N_{i}^{\prime}\left[a_{j} ; a_{i} \rrbracket * Q \llbracket a_{i} \cdot s_{Q} \rrbracket * N_{j}^{\prime}\left[s_{Q} ; b_{j} \rrbracket * Q \llbracket b_{j} ; t \rrbracket\right.\right.$ traverse the edges of $Q \llbracket s_{Q} ; a_{i} \rrbracket$ in either directions. The subpath $Q \llbracket b_{i} ; t_{Q} \rrbracket$ is treated in a similar manner.

- CASE. $3 s=a_{i}$ and $t$ is an internal vertex of the quadrant II. Let $J[s ; t]$ in quadrants I and II be $P \llbracket s ; t \rrbracket$ and $Q \llbracket s ; t \rrbracket$ be its complement in $J$. We may assume that $s_{P}=a_{j}$ and $s_{Q}=b_{j}$. Since, otherwise, we can reduce this case to an instance of the case(1) or case(2)

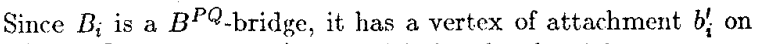
$P \llbracket s_{P} ; t_{P} \|$. Let $b_{i}$ and $b_{i}^{\prime}$ be modified to be the right-most vertices of attachment of $B_{i}$ on $P$ and $Q$, distinct from $t$. Accordingly $N_{i}$ is modified. Moreover, there are cross-cuts between $a_{i}$ and $b_{i}^{\prime}$ (say, $N_{i}^{\prime}$ ) and between $b_{i}$ and $b_{i}^{\prime}$ (say, $N_{i}^{\prime \prime}$ ). Observe that $t_{Q}$ is distinct from $b_{i}$ if and only if $t_{Q}$ is the right-most vertex of attachment of $B_{j}$ distinct from $t$. A similar relation holds between $t_{F}$ and $b_{i}^{\prime}$.

Every edge of the residual path $Q \llbracket b_{j} ; b_{i} \llbracket$ is 'bidirectional'. The paths $Q \llbracket s ; t \rrbracket$ and $N_{i} \llbracket s ; b_{i} \rrbracket * Q \llbracket b_{i} ; b_{j} \rrbracket * N_{j} \llbracket b_{j} ; a_{j} \rrbracket * Q \llbracket a_{j} ; t \rrbracket$ traverse the edges of $Q\left[b_{j} ; b_{i}\right]$ in cither directions. If $a_{j}$ and $b_{i}^{\prime}$ are distinct then, similarly, every edge of $P \llbracket a_{j} ; b_{i}^{\prime} \rrbracket$ is 'bidirectional'.

If $t_{P}$ and $b_{i}^{\prime}$ are distinct then every edge of $P \llbracket b_{i}^{\prime} ; t_{P} \llbracket$ is 'bidirectional'. Since $t_{P}$ is a vertex of attachment of $B_{j}$, there is a cross-cut between $a_{j}$ and $t_{P}$ (say, $N_{j}^{\prime}$ ). The paths $P \llbracket s ; t \rrbracket$ and $I^{\prime} \llbracket s ; a_{j} \rrbracket * N_{j}^{\prime} \llbracket a_{j} ; t_{P} \rrbracket * P \llbracket t_{P} ; b_{i}^{\prime} \rrbracket * N_{i}^{\prime \prime} \llbracket b_{i}^{\prime} ; b_{i} \rrbracket * Q \llbracket b_{i} ; t \rrbracket$ traverse edges of $P \llbracket b_{i}^{\prime} ; t_{P} \rrbracket$ in either directions. Similarly, for $Q \llbracket b_{i} ; t_{Q} \rrbracket$.

- CASE.4 $s=n_{i}$ and $t=b_{i}$. We may assume that $s_{P}=t_{P}=$ $a_{j}$ and $s_{Q}=t_{Q}=b_{j}$. Since, otherwise, we can reduce this case to an instance of the case $(3)$. Since the pathis are empty, the theorem is trivially true in this case.

Theorem 3.11: Assume LABEL-TYPE-III correctly labels the edges of a TYPE.III graph. Then the algorithm LABEL-TYPE-II correctly labels a TYPE.II graph.

PROOF. Let $e$ be an arbitrary edge of the TYPE. III graph $G$. Without loss of generality we may assume that $e$ is an edge of the path $P$, and that there are no two intcrlacing bridges such that $e \in P\left[s_{P} ; t_{P}\right]$, as in the previous lemma. Otherwise the proof is immediate from the previous two lemmas.

We partition the set of $B^{P Q}$-bridges, $B$ of $J$ into following three disjoint subsets: $B_{1}=$ the set $B^{P Q}$-bridges whose vertices of attachmont on $P$ are to the left of $e ; B_{2}=$ the set $B^{I-2}$-bridges whose vertices of attachment on $P$ are to the right of $e ; B_{3}=$ $B \backslash\left\{B_{1} \cup B_{2}\right\}$. Notice that no bridge of $B_{i}$ interlaces with a bridge of $B_{j}$, (where $i, j=1,2,3$ and $i \neq j$ ); and $B_{3}$ docs not contain a pair of interlacing bridges. If $B_{3}$ is empty or if $B_{3}$ contains

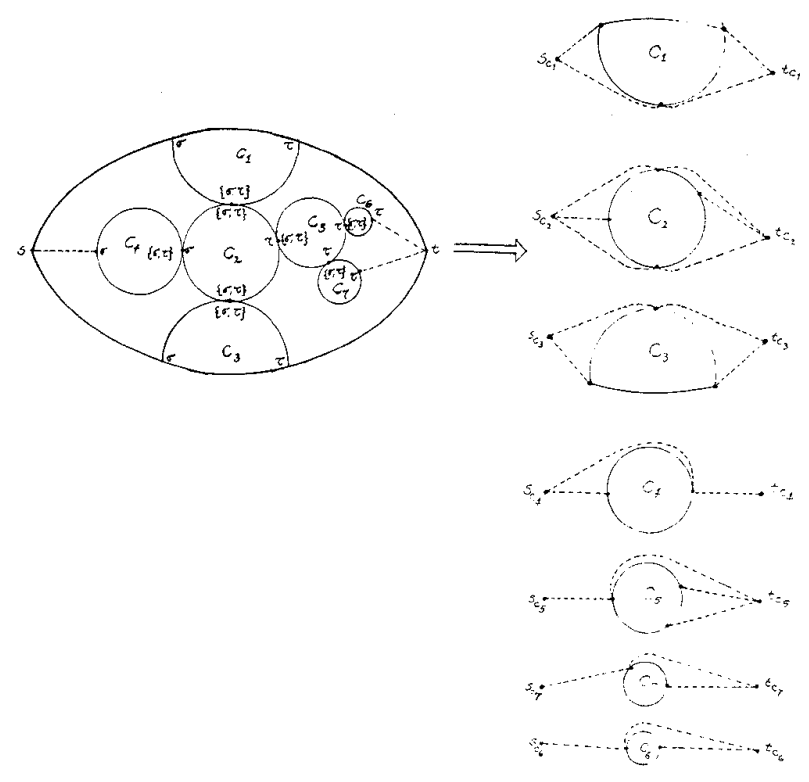

Figure 8: Steps of the Algorithm LABEL-TYPE-III.

two or more equivalent $B^{P Q}$ 3-bridges (Cf. Proposition 1.13) then it can be shown that $\ell(e)=\langle u, v\rangle$, if $u$ is to the left of $v$ on $P$. On the other hard, if $B_{3}=\left\{B_{3}\right\}$ is a singleton then the labelling of $e$ is completely determined by the bridge fragment $J \cup B_{3}$.

\subsection{Labelling a Type.III Graph}

We assume that there is an algorithm called LABELTYPE-IV, to label the edges of a TYPE.IV graph.

Algorithm LABEL-TYPE-III. (Cf. Figure 8.)

-STEP 1. Let $s_{F}$ and $t_{P}$ be the left- and right-most vertices of attachment of $B$ on $P$; and similarly, $s_{Q}$ and $t_{Q}$, on $Q$. Let $G^{\prime}$ be the subgraph derived from $G$ by deleting the vertices $s$ and $t$ together with the edges incident on $s$ and $t$ and the subpaths $P \llbracket s ; s_{P} \rrbracket, Q \llbracket s ; s_{Q} \rrbracket, P \llbracket t_{P} ; t \rrbracket$ and $Q \llbracket t_{Q} ; t \rrbracket$. The vertices $s_{P}, s_{Q}$ and the vertices of $G^{\prime}$ adjacent to $s$ in $G$ are labelled $\sigma$; and the vertices $t_{P}, t_{Q}$ and the vertices of $G^{\prime}$ adjacent to $t$ in $G$ are labelled $\tau$.

-STEP2. Find the nonseparable components of $G^{\prime}$ and label each separation vertex $v$ of each nonseparable component $C$ as follows: $v$ is labelled $\sigma$ (respectively, $\tau$ ), if there is a path from $v$ to a vertex $u$, already labelled $\sigma$ (respectively, $\tau)$, in the tree $T\left(G^{\prime}\right)$ of $G^{\prime}$ and the path avoids $C$. This step can be done in time $O\left(\operatorname{size}\left(T\left(G^{\prime}\right)\right)\right)=O(|E|)$ using a depth first search.

-STEP 3. For each nonseparable component $C$, introduce two new vertices $s_{C}$ and $t_{C}$; and join $s_{C}$ (respectively, $t_{C}$ ) to all the vertices of $C$ labelled $\sigma$ (respectively, $\tau$ ). We call the graph derived from the nonseparable component $C, G_{C}$; and the new edges, pseudo-edges (III). If $G^{\prime}$ is 
nonscparable then the graph derived from the nonsemarable component $G^{\prime}$, called $G^{\prime \prime}$, is a TYPE.IV graph and by assumption, we can label $G^{\prime \prime}$ using the algorithm LABELTYPE-IV. Otherwise, label the edges of the components by recursively calling the main algorithm with $\left(G_{C ;} ; s_{C}, t_{C}\right)$.

-STEP4. Edge e $=[u, v]$ on $P\left[s ; s_{P} \rrbracket, Q \llbracket s_{s} ; s_{Q} \rrbracket, P \llbracket\left[t_{P} ; t \rrbracket\right.\right.$ and $\left.Q \| t_{Q} ; t\right]$ is labelled $\ell(e)=\langle u, v\rangle$, if $u$ is to the left of $v$ on $P$ or $Q$. Edge $[s, u]$ incident on $s$ is labelled $\ell([s, u])=$ $\langle s, u\rangle$ and edge $[u, t]$ incident on $t$ is labelled $\ell([u, t])=$ $\langle u, t\rangle$.

Lemma 3.12: Let $C$ be a nonseparable component of $G^{\prime}$ whose separation vertices are labelled $\sigma$ or $\tau$ (or both), and let $e$ be an arbitrary edge of $C$. Then there is a simple path $R$ from $s$ to $t$ in $G$ traversing $e$ in the order $u$, $v$ if and only if there are two distinct separation vertices $a$ and $b$ in $C$ such that (i) Label of $a$ is $\sigma$; and label of $b$ is $\tau$. (ii) There is a simple rath $R^{\prime}$ joining $a$ and $b$ in $C$; and $R^{\prime}$ traverses $e$ in the sume order.

PROOF. $(\Leftrightarrow)$ By labelling of the spparation vertices in the tree $T\left(G^{\prime}\right)$ of $G^{\prime}$, there are simple path $N_{1} \llbracket s ; a \rrbracket$ and $N_{2} \llbracket b ; t \rrbracket$ in $G$ such that $N_{1}$ and $N_{2}$ are vertex disjoint and do not belong to $C$. The path $R \llbracket s ; t \rrbracket=N_{1} \llbracket s ; a \rrbracket * R^{\prime} \llbracket a ; b \rrbracket * N_{2} \llbracket b ; t \rrbracket$ in $G$, is simple and traverses in the same order as $\left.R^{\prime} . \Leftrightarrow\right)$ Let $R \llbracket s ; t \rrbracket$ traverse $e$ in the order $u$ and $v$ and let $e$ belong to the nonseparable component $C$. Then $R \llbracket s ; t \rrbracket$ can be written as $R \llbracket s ; a \rrbracket * R \llbracket a ; b] *$ $R \llbracket b ; t \rrbracket$, where $a$ and $b$ are two distinct separation vertices of $C$. Let $R^{\prime}$ in $C$ be the subpath $\left.R \llbracket a ; b\right]$. $R^{\prime}$ traverses the edge $e$ in the same order as $R$ in $G$. Moreover, the path $R \llbracket s ; a \rrbracket$ induces a path in the tree $T\left(G^{\prime}\right)$ such that it joins a to some vertex lahelled $\sigma$ and the path avoids $C$. Hence the separation vertex is labelled $\sigma$. Similarly the separation vertex $b$ is labelled $\tau$.

Theorem 3.13: Assume that LABEL-TYPE-IV correctly labels the edges of a TYPE.IV $g$ aph.' Then the algorithm LABEL-TYPE-III correctly labels the edges of a TYPE.II graph.

PROOF. By induction on the size of the graph and the Lemma 3.12 .

\subsection{Labelling a Type.IV Graph}

Theorem 3.14: Suppose we have an Algorithm LABELTYPE-IV that correclly labels the edges of a TYPE.IV graph in time $O(T(|E|,|V|)) \geq O(|E| \cdot|V|)$, where $T$ $i$ s a monotonic nondecreasing function in $|E|$ and $|V|$. Then the set of mutually recursive algorithms, LABELGRAPH, LABEL-TYPE-I, LABEL-TYPE-IT and LABELTYPE-III, correctly labels the edges of an undirected connected strict graph in time $O(T(|E|,|V|)+|E| \cdot|V|)$

PRoOF. (1) Follows immeciately from the Theorems 3.5, 3.8, 3.11 and 3.13 .

(2) The set of mutually recursive algorithms works as a Divideand-Conguer algorithm: and cach divide step and conquer step takes $O(|E|+|p L|$, where $|p E|=$ the number of pseudo-edges introduced at cach stage. Since cach 'divide' step reduces the munber of vertics of the subgraph by at least one, there can be at most $O(|V|)$ stages of 'divide' stages before the graph is diviked into a set of TYPE.IV graphs.

Claim. The total number of pscudo-edges in all the subgraphs that are produced at the end of each stage, $|p E|=O(|E|)$.

Proof of the Claim. Let $G^{\prime}$ be a subgraph produced at some stage. We define three functions $f_{1}, f_{2}$ and $f_{3}$ such that $f_{2}$ maps at most four distinct pseudo-edges(II) of $G^{\prime}$ to exactly one graph-eclge, $f_{3}$ maps at most four distinct pseudo-edges(III) to exactly one graph-edge, and $f_{1}$ is an injective function mapping a pseudo-edge(I) to a graph-edge or a pseudo-cdge(III). Since the graph-edges of the subgraphs are disjoint, the claim follows.

If $\epsilon^{\prime}$ is a pseudo-edge, incident on a vertex $v$ of $G^{\prime}$ then there must be $B^{P Q_{-}}$bririge of $G^{\prime}$ with a vertex of attachment at $v$. Let $e$ be the edge of the $B^{P Q}$-bridge, incident at $v$. Define $f_{2}\left(e^{\prime}\right)=e$. Since $\int_{2}$ maps at vost four distinct pseudo-edges(II) of $G^{\prime}$ to one graph-edge of $G^{\prime}$, and since the graph-edges of the subgraphs are disjoint, the total number of pseido-edges(II), $\left|p E^{\mathrm{II}}\right| \leq 4 \cdot|E|$. If $e^{\prime}$ is a pseudo-edge(III), incident at a vertex $v$ of $G^{\prime}$, then since $v$ is one of $s_{P}, t_{P}, s_{Q}, t_{Q}$, a vertex adjacent to $s$ or $t$, or a separation vertex of a componint then there must be graphedge $e$ that is also incident on $v$. Define $f_{3}\left(e^{\prime}\right)=e$. Since $f_{3}$ maps at most fou? pseudo-edges(III) of $G^{\prime}$ to exactly one graphedge, the total number of pseudo-edges(III), $\left|p E^{\Pi I}\right| \leq 4 \cdot|E|$. We define a injective function $f_{1}$ that maps a pseudo-edge(I) to a graph-edge or a pseudo-edge(III). Let $\mathbf{B}_{i}$ be a block with the associated vertices $s_{i}$ and $t_{i}$ and $J_{i}$, the ambitus of $\mathbf{B}_{i}$, containing both $s_{i}$ and $t_{i}$. Let $e^{i}=\left[s_{i}, t_{i}\right]$ be the pseudo-edge(I) and let $e$ be an edge of $J_{i}$ incident on $t_{i}$ but not belonging to $J$. Define $f_{1}\left(e^{\prime}\right)=e$. It is easy to show that $f_{1}$ is one-one and hence, the total number of pscudo-edges(I), $\left|p E^{\mathrm{Y}}\right| \leq 5 \cdot|E|$. Summing the number of pseudo-edges, we obtain $\left|p E^{\mathrm{I}}\right|+\left|p E^{\mathrm{II}}\right|+$ $\left|p E^{\mathrm{III}}\right| \leq 13 \cdot|E| . \quad$ (End of the Claim.)

Hence, the algorithms spend $O(|E| \cdot|V|)$ time to rednce the graph $G$ into set of TYPE.IV sulyraphs. Since cach TYPE. IV subgrapl has less than $|V|$ vertices, and since the total number of edoes is $O(|E|)$, the theorem follows.

Theorem 3.15: Let $G$ be a TYPE.IV graph and $G^{\prime}$, the subgraph derived from $G$ by deleting s and together with their incident edges. Let $B$ be the $B^{P Q}$-bridge of $G$. Then every edge $e$ of $B$ not incident on $s$ or $t$ is 'bidirectional.'

PROOF. Observe that there exist vertices of attachunent of $B, x$ on $P \llbracket s ; t \llbracket$ and $y$ on $Q \rrbracket s ; t \llbracket$ such that there is a cross-cut $N$ between $x$ and $y$ containing $e$. (This is a consequence of proposition 1.12 and the nonseparability property of $G^{\prime}$.) The two paths $P \llbracket s ; x \rrbracket * N \llbracket x ; y \rrbracket * Q \llbracket y ; t \rrbracket$ and $Q \llbracket s ; y \rrbracket *(N \llbracket x ; y \rrbracket)^{\mathrm{R}_{*}}$ $P \llbracket x ; t$ itraverse $e$ in either directions.

Every edge $[s, u]$ incident on $s$ is labelled $\langle s, u\rangle$ and every edge $[u, t]$ incident on $t$, labelled $\langle u, t\rangle$. All other edges of the $B^{P Q}$-bridge are labelled 'bidirectional.' Hence, we can label all but the edges on $P$ and $Q$ of a TYPE.IV graph in $O(|E|)$ time. But, each edge of $P \llbracket s_{P} ; t_{P} \rrbracket$ and $Q \llbracket s_{Q} ; t_{Q} \rrbracket$ can be labelled in $O(|E| \cdot|V|)$ time, asing an algorithm for Two-Disjoint-Path problem. Since there are at most $|V|$ such edges, this step can be done in $O\left(|E| \cdot|V|^{2}\right)$ time, thus, yielding an $O\left(|E| \cdot|V|^{2}\right)$ labelling algorithm for the general graph. 


\section{An Efficient Algorithm.}

We sketch an algorithm to label the edges of the sulpath $P \llbracket s_{p} ; t_{P} \rrbracket$ and $Q \llbracket s_{Q} ; t_{Q} \rrbracket$ of a TYPE.IV graph, $G$, in $O(|E| \cdot|V|)$ time. Recall that this will provide an $O(|E| \cdot|V|)$ time algorithm for a general graph. (Cf. Theorems 3.14.) The algorithm makes use of many special properties of a bridge and its proofs of correctness are rather complicated. These will be supplied in the author's thesis.

\subsection{U-Fragment}

Before we describe the main algorithm, we introduce the notion of a U-Fragment and sketch an algorithm to find certain pairs of disjoint cross-cuts in it.

Definition 4.1: A U-Fragment and $\bar{U}$-Fragmert are defined inductively on the structure of a TYPE.II graph as follows:

-A TYPE.II graph is called a U-Fragment, with $B_{2}=$ its

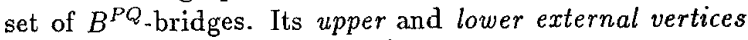
of attachment are empty sets.

- Let $U$ be a U-Fragment consisting of the circuit $J=$ $P \cup Q$ and let $B$ be a $B^{P Q}$-bridge of $U$. Let the left-most and the right-most vertices of attachment of $B$ on $Q \llbracket s ; t \rrbracket$ be the distinct vertices ' $s^{\prime}$ and $t^{\prime}$. Let $R$ be a path in $B$, connecting $s^{\prime}$ and $t^{\prime}$ and decomposing $B$ into following sets of bridges with respect to $\{P\} \cup\{Q\} \cup\{R\}: B_{1}=$ =the set of bridges with vertices of attachment on $P \rrbracket s ; t \llbracket$ and on $R \rrbracket s^{\prime} ; t^{\prime} \llbracket ; B_{2}=$ the set of bridges with vertices of attachment on $R \rrbracket s^{\prime} ; t^{\prime} \llbracket$ and $Q \rrbracket s^{\prime} ; t^{\prime} \llbracket ;$ and the set of bridges with vertices of attachment solely on $R$ and avoiding $B_{1}$ and $B_{2}$.

The subgraph $U^{\prime}=\left\{R \llbracket s^{\prime} ; t^{\prime} \rrbracket\right\} \cup\left\{Q \llbracket s^{\prime} ; t^{\prime} \rrbracket\right\} \cup B_{2}$ of $U$, is called a $U$-Fragment of $U$ on $Q$. The vertices of attachment of $B_{1}$ on $R \rrbracket s^{\prime} ; t^{\prime} \llbracket$ are called its upper external vertices of attachment, $U A$ and the lower external vertices of attachment of $U$ lying on $Q \rrbracket s^{\prime} ; t^{\prime} \llbracket$, its lower external vertices of attachment, LA.

The subgraph $\bar{U}^{\prime}=\left\{R \llbracket s^{t} ; t^{\prime} \rrbracket\right\} \cup\{P \llbracket s ; t \rrbracket\} \cup B_{1}$ of $U$, is called a complementary U-Fragment (simply $\overline{\mathrm{U}}$-Fragment) of $U$ on $Q$. The external vertices of attachment of $U$ lying on $P \llbracket s ; t \llbracket$ are called its upper external vertices of attachment, $U A$, and the vertices of attachment of $B_{2}$ on $R \rrbracket s^{\prime} ; t^{\prime} \|$ together with the vertex $s^{\prime}$ (if $s^{\prime}$ is distinct from $s$ ) and the vertex $t^{\prime}$ (if $t^{\prime}$ is distinct from $t$ ), its lower external vertices of attachment, LA.

The U- and $\bar{U}$-Fragments of an $\bar{U}$-Fragment are defined in an identical manner.

NOTATION: Let $U$ be a $U$ - or a U-Fragment. Let the left- and right-most upper attachments on $P$ (respectively, $Q$ ) be $s_{U}$ and $t_{U}$ (respectively, $s_{L}$ and $t_{L^{*}}$ ) Let $B$ be a $B^{P Q_{\text {-bridge }}} U$ and let the left- and right-most vertices of attachment of $B$ on $P \llbracket s ; t \rrbracket$ be $s_{P}^{B}, t_{P}^{B}$, and those on $Q \llbracket s ; t \rrbracket$ be $s_{Q}^{B}$ and $t_{Q}^{D}$. Similarly, let the left- and right-most vertices of attachment of $\dot{B}$ on $P \rrbracket s ; t[$ be
$s_{P}$ and $t_{P}$, and those on $Q \rrbracket s ; t$ [ be $s_{Q}$ and $t_{Q}$. We consider the bridge $B$ angmented with the paths as follows: Let the modified bridge be $B \cup P \llbracket s_{P}^{B} ; t_{P}^{B} \rrbracket \cup Q \llbracket s_{Q}^{B} ; t_{Q}^{B} \rrbracket$. If $s_{P}^{B}=s_{Q}^{B}=s$ then label the vertex with ' $s$ ', and if $t_{P}^{D}=t_{Q}^{B}=t$ then label the vertex with ' $t$.' By an abuse of notation, we also refer to the modified bridge as the bridge, $B$.

Definition 4.2: A U-Fragment, $U^{\prime}$, of $U$ (a U- or a $\bar{U}$ Fragment) on $Q$ is said to be a Feasible U-Fragment, if it satisfies at least one of the following two conditions:

1. $|\mathrm{LA}|>0$ and $|\mathrm{UA}|>0$.

2. (i) $|\mathrm{L} \mathrm{A}|=0$; (ii) not all the vertices of attachment of $B_{2}$ on $P^{\prime}$ belong to $P^{\prime} \rrbracket s^{i} ; s_{U} \rrbracket$ or to $P^{\prime} \llbracket t_{U} ; t^{\prime} \llbracket ;$ and (iii) there exist two vertex disjoint paths in $B_{1}$, $R_{a} \llbracket a^{\prime} ; a \rrbracket$ and $R_{b} \llbracket b^{\prime} ; b \rrbracket$, where $R_{a}\left(R_{b}\right)$ meets $P \rrbracket s ; t \llbracket$ only in $a(b)$ and meets $\left.P^{\prime}\right] s^{\prime} ; t^{\prime}\left[\right.$ only in $a^{\prime}\left(b^{\prime}\right)$.

Definition 4.3: A U-Fragment, $\bar{U}^{\prime}$, of $U$ (a U- or a $\overrightarrow{\mathrm{U}}-$ Fragment) on $Q$ is said to be a Feasible $\bar{U}$-Fragment, if $|\mathrm{LA}|>0$ or $|\mathrm{UA}|>0$.

Henceforth, it will be implicitly assumed that U-Fragment and U-Fragment are found using the following conventions:

Convention 4.4: Let $U^{\prime}$ be a U- or $\overline{\mathrm{U}}$-Fragment with the $B^{P Q}$-bridge $B$.

$-s_{P}^{B}=t_{P}^{B}$ and $s_{Q}^{B}=t_{Q}^{B}$. Then $B$ may be discarded.

- $s_{P}^{B}=t_{P}^{B}$ and $s_{Q}^{B}$ and $t_{Q}^{B}$ are distinct. If there is a lower vertex of attachment $b \in Q^{\prime} \rrbracket s_{Q}^{B} ; t_{Q}^{B} \llbracket$ then the U- and $\overline{\mathrm{U}}$ Fragment in $B$ are on $Q$. Otherwise, $B$ is discarded.

- $s_{P}^{B}$ and $t_{P}^{B}$ as well as $s_{Q}^{B}$ and $t_{Q}^{B}$ are distinct. (a) ( $U^{\prime}$ is a U-Fragment satisfying 1 . of definition 4.2.) If $B$ has no lower attachment on $Q^{\prime} \rrbracket s_{Q}^{B} ; t_{Q}^{B} \llbracket$, but has an upper attachment on $P^{\prime} \rrbracket s_{P}^{B} ; t_{P}^{B} \llbracket$ then the U- and $\overline{\mathrm{U}}$-Fragment in $B$ are on $Q$. Similarly, with $P$ and $Q$ interchanged. $(b)\left(U^{\prime}\right.$ is a U-Fragment of $U$ on $Q$ satisfying 2. of definition 4.2.) The U- and $\bar{U}$-Fragment in $B$ are on $Q$. (c) (U' $U^{\prime}$ is a $\bar{U}$ Fragment of $U$ on $Q$.) The U- and $\overline{\mathrm{U}}$-Fragment in $B$ are on $Q$. Otherwise, the U- and $\bar{U}$-Fragment are on $P$ or $Q$, the choice being arbitrary.

Definition 4.5: (Cf. Figure 9.) Let $U$ be a U-Fragment. -Two vertex-disjoint cross-cuts $N_{1} \llbracket x_{P} ; x_{Q} \rrbracket$ and $N_{2} \llbracket y_{P} ; y_{Q} \rrbracket$ are said to be a PQ-Cross-Cut Pair, if: (1) $x_{P}, y_{P} \subseteq$ $P \rrbracket s ; t \llbracket$ and $x_{P}$ is to the left of $y_{P} ;$ (2) $x_{Q}, y_{Q} \in Q \rrbracket s ; t \llbracket$ and $x_{Q}$ is to the right of $y_{Q}$ and (3) if $|\mathrm{LA}|=0$ or if $x_{Q}, y_{Q} \in Q \rrbracket s ; s_{L} \rrbracket$ then $|\mathrm{UA}|>0$ and not both $x_{P}$ and $y_{P}$ belong to $P \llbracket s ; s_{U} \rrbracket$; and similarly, if $|\mathrm{LA}|=0$ or if $x_{Q}$, $y_{Q} \in Q \llbracket t_{L} ; t \mathbb{I}$ then $|\mathrm{UA}|>0$ and not both $x_{P}$ and $y_{P}$ belong to $P \llbracket t_{U} ; t \llbracket$. (Or, with UA and LA interchanged.)

- Two vertex-disjoint cross-cuts $N_{1} \llbracket s ; t \rrbracket$ and $N_{2} \llbracket x_{P} ; x_{Q} \rrbracket$ are said to be an $S T$-Cross-Cut Pair, if: (1) $x_{P} \in P \rrbracket s ; t \rrbracket$ and $x_{Q} \in Q \rrbracket s ; t \llbracket ;(2)$ there are an upper attachment on $P \rrbracket s ; t[$ and a lower attachment on $Q \rrbracket s ; t \llbracket$.

-Two vertex-disjoint cross-cuts $N_{1} \llbracket x_{P}^{\prime} ; x_{P}^{\prime \prime} \rrbracket$ and $N_{2} \llbracket y_{P} ; y_{Q} \rrbracket$ are said to be a P-Cross-Cut Pair, if: $(1) x_{P}^{\prime}, x_{P}^{\prime \prime} \in P \llbracket s ; t \rrbracket$ and at least one of them is distinct from $s$ and $t ;(2)$ 


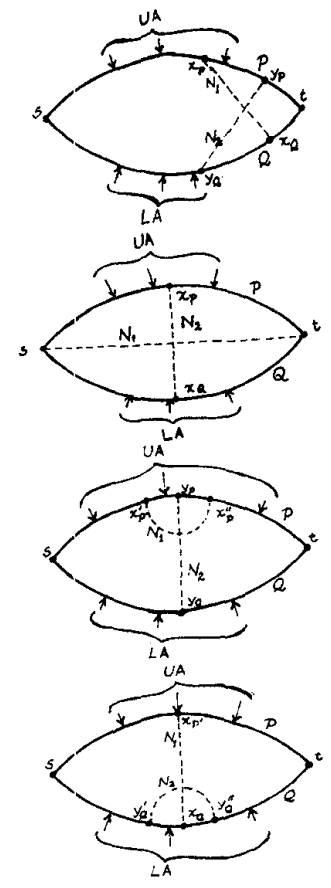

Figure 9: $P Q-, S T \cdot, P$ - and $Q$-Cross-Cut Pairs.

$y_{P} \in P\left\|x_{P}^{\prime} ; x_{P}^{\prime \prime}\right\|$ and $y_{Q} \in Q \| s ; t \llbracket ;$ and $(s)$ there is an upper attachment $c^{\prime} \in P \rrbracket x_{P}^{\prime} ; x_{P}^{\prime \prime} \llbracket$ :

A Q-Cross-Cut-Pair is defined similarly.

The Cross-Cut Pairs of U-Fragments and bridges are defined similarly. Now, we present an algorithm to find a $P_{-}, Q_{-}, P Q_{-}$or $S T$-Cross-Cut Pair in a U-Fragment.

\section{Algorithm ANALYZE-U-FRAGMENT.}

-STEP1. First, check the interlacing bridges of $B_{2}$ to determine if the required cross-cut pairs exist. If so, return 'YES'. In the next step, analyze each bridge of $B_{2}$ for the cross-cut pairs. If the answer is 'YES' for any $B_{2}$ bridge, return 'YES'; otherwise, return 'NO'.

-STEP2. Let $B \in B_{2}$. If the vertices of attachment of $B$ on $P$ belong to $P \rrbracket s ; s_{U} \rrbracket$ and those on $Q$ belong to $Q \rrbracket s ; s_{L} \rrbracket$ (or symmetrically, if those on $P$ belong to $P \llbracket t_{U} ; t \llbracket$ and those on $Q$ belong to $Q \llbracket t_{L} ; t \llbracket$ then $B$ can not have the required cross-cut pairs and is discarded. Otherwise, analyze each single bridge using the algorithm ANALYZE-BRIDGE.

\footnotetext{
Algorithm ANALYZE-BRIDGE $(B)$ :

begin

DIVIDE : Find a path $R$ in $B$ following the convention 4.4.

Modify $R$ such that the bridges with vertices of attachment solely on $R$ avoid other bridges. Let $B_{1}$ and $B_{2}$ be as in the definition 4.1 and let $B_{3}$ be the set of bridges with vertices attachment on $P \llbracket s_{F}^{E} ; t_{P}^{B} \rrbracket, R \rrbracket s_{Q}^{B} ; t_{Q}^{B} \rrbracket$ and $Q \rrbracket s_{Q}^{B} ; t_{Q}^{B} \rrbracket$ if TEST-AND-MODIFY $(B)$ returns 'YES' then if $\left|B_{3}\right| \neq 0$ then return 'YES' else goto RECUR; else goto UBAR;
}

RECUR :Let $U$ be the U-Fragment formed by the paths $P$, $R$ and the bridges $B_{2}$.

if $U$ is marked feasible then

if ANALYZE-U-FRAGMENT(U) returns 'YES' then returu 'YES';

UBAR :[Analyze the set of $B_{1}$-bridges:]

Analyze bridges $B_{1}$ to determine if it has the required

Cross-Cut pairs. If so, return 'YES'.

[Analyze each bridge $B^{\prime} \in B_{1}:$ ]

Let the left- and right-most lower attachments of the $\bar{U}$ -

Fragment on $R$ be $s_{L}^{\prime}$ and $t_{L}^{\prime}$.

If the vertices of attachment of $B^{\prime}$ on $P$ belong to $\left.P \backslash s ; s_{U}\right]$ and those on $R$ belong to $R \rrbracket s ; s_{L}^{\prime}$ ! (or symmetrically, if those on $P$ belong to $P \| t_{U} ; t \llbracket$ and those on $R$ belong to $\left.R \llbracket t_{L}^{\prime} ; t \llbracket\right)$ then discard $B^{\prime}$;

if ANALYZE- BRIDGE $\left(B^{\prime}\right)$ returns 'YES' then return 'YES';

return 'NO';

end .

\section{Algorithm TEST-AND-MODIFY.}

-STEP1. Check if $B$ has a lower vertex of attachment on $Q \rrbracket s_{Q}^{B} ; t_{Q}^{B}$. Then, if $\left|B_{3}\right| \neq 0$ then return 'YES'. If $\left|B_{3}\right|=0$ then mark $U$ 'feasible' and return 'YES'. Otherwise, go to the next step.

-STEP 2. Let the left- and right-most vertices of attachment of $B_{1}$ and $B_{3}$ bridges on $R$ be $s_{R}^{\prime}$ and $t_{R}^{\prime}$ (and if $\left|B_{3}\right|=0$ then those on $Q \| s_{Q}^{B} ; t_{Q}^{B} \llbracket$ be $s_{Q}^{\prime}$ and $t_{Q}^{\prime}$.) Let $G_{B}$ be the subgraph of $B$ obtained by deleting the followings: $(i)$ the bridges $B_{2}$, (ii) the vertices $s_{Q}^{B}$ and $t_{Q}^{B}$ together with the edges incident on them, (iii) the subpaths $R \llbracket s_{Q}^{B} ; s_{R}^{\prime} \rrbracket$ and $R \llbracket t_{R}^{\prime} ; t_{Q}^{B} \rrbracket,(i v)$ the subpath $Q \llbracket s_{Q}^{B} ; t_{Q}^{D} \rrbracket$, if $\left|B_{3}\right|=0$ (or the subpaths $Q \llbracket s_{Q}^{D} ; s_{Q}^{\prime} \rrbracket$ and $Q \llbracket t_{Q}^{\prime} ; t_{Q}^{B} \rrbracket$, if $\left|B_{3}\right| \neq 0$.) The vertices of $G_{B}$ adjacent to $s_{Q}^{B}$ are labelled $\sigma$; those adjacent to $t_{Q}^{B}, \tau$; the vertices $s_{R}^{\prime}$ and $t_{R}^{\prime}, \rho$; and the vertices $s_{Q}^{\prime}$ and $t_{Q}^{\prime}, \chi$.

-STEP 3. Find the nonseparable components of $G_{B}$ and let the component containing the path $P \llbracket s_{P}^{B} ; t_{P}^{B} \rrbracket$ be called $C_{\pi}$. Let each separation vertex of $C_{\pi}$ be labelled as follows: the separation vertex $v$ is labelled $\sigma$ (respectively, $\tau, \rho$ or $\chi$ ) if there is a path from $v$ to a vertex $u$, already labelled $\sigma$ (respectively, $\tau, \rho$ or $\chi$ ) in the tree $T\left(G_{B}\right)$ of $G_{B}$ and the path avoids $C_{\pi}$.

-STEP 4 If $\left|B_{3}\right| \neq 0$ and $C_{\pi}$ has two distinct vertices one labelled $\rho$ and the other labelled $\chi$ then return 'YES'. If $\left|B_{3}\right| \doteq 0$ and $C_{\pi}$ has two distinct vertices labelled $\rho$ then check if $U$ satisfies $2($ ii) of definition 4.2. If so, mark $U$ 'feasible' and return 'YES'.

Otherwise, modify the bridge $B$ to form a $\bar{U}$-Fragment as follows: Delete all the edges of the bridge except the ones in $C_{\pi}$; Adjoin the separation vertex of $C_{\pi}$, labelled $\rho$, to $s_{Q}^{B}$ and $t_{Q}^{B}$ with new edges; And adjoin the separation vertices of $C_{\pi}$, labelled $\sigma$ (respectively, $\tau$ ) to $s_{Q}^{\mathrm{B}}$ (respectively, $\left.t_{Q}^{B}\right)$. Let the path from $s_{Q}^{B}$ to $t_{Q}^{B}$, touching the vertex labelled $\rho$, be $R$ and $B_{1}{ }^{\prime}$, the set of bridges with vertices of attachment only on $P$ and $R$. Return the modified graph as the $\bar{U}$-Fragment. 


\subsection{Labelling the Path}

We present an algorithm to label the path $P$ of a TYPE.IV graph, $G$, in $O(|E| \cdot|V|)$ time. By using the algorithm twice (once for $P$ and once for $Q$ ), it is possible to label the paths of $G$ in $O(|E| \cdot|V|)$ time. In this section, we only sketch the algorithm for the case when the bridge $B$ of $G$ has its vertices of attachment $s_{Q}^{B}$ and $t_{Q}^{B}$ distinct from each other and from $s$ and $t$. The other cases are similar, but slightly more complicated; and will appear in the full paper.

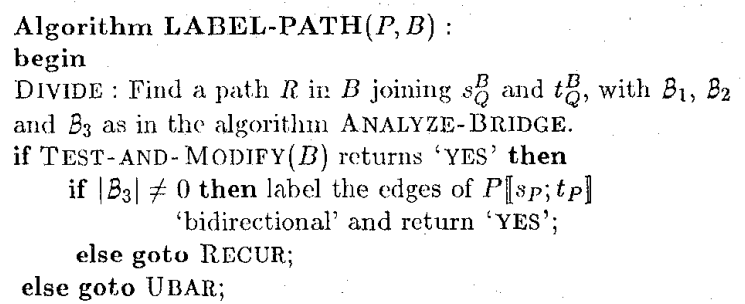

RECUR :Let $U$ be the U-Fragment as in ANALYZE-BMIDGE if $U$ is marked feasible then

if ANALYZE-U-FRAGMENT $(U)$ returns 'YES' then label the edges of $P \llbracket s_{P} ; t_{P} \rrbracket$ 'bidirectional' and return 'YES';

UBAR :[Analyze the set of $B_{1}$-bridges:]

Analyze the blocks of interlacing bridges of $\dot{B}_{1}$ to label the edges of the path $P \llbracket s_{P} ; t_{P} \rrbracket$, as in LABEL-TYPE-II; Also, determine if they have the required Cross-Cut pairs. If so, label the edges of $P \llbracket s_{P} ; t_{P} \rrbracket$ 'bidirectional' and return 'YES';

[Analyze each bridge $B^{\prime} \in B_{1}$ :

-. - We analyze each bridge $B^{\prime}$ to label the subpaths

- $P \llbracket s_{P}^{B^{\prime}} ; t_{P}^{B^{\prime}} \rrbracket$ (recursively), as well as to find if it has the

- required cross-cut pairs, using LABEL-PATH,

if LABEL-PATH $\left(P, B^{\prime}\right)$ returns 'YES' then

label the edges of $P \llbracket s_{P} ; t_{P} \llbracket$ 'bidirectional' and return 'YES';

return 'NO';

end .

Notice that the algorithm uses Divide-and-Conquer paradign: Each 'divide' step involves finding a path in a bridge, where the path has additional properties that the bridges with vertices of attachment solely on the path avoid $B_{1}, B_{2}$ and $B_{3}$. This can be done in $O(|E|+|p E|)$ time by combining DFS technique with the ambitus-findin: algorithm. ( $p E=$ the pseudo-edges introduced in the step 4 of TEST-AND-MODIFY.) Each 'conquer' step involves analyzing intcrlacing bridges for appropriate crosscut pairs, which can be done in linear time, and analyzing each individual bridge (recursively). However, each 'divide' step reduces the number of vertices of the subgraph by at least one, and hence the algorithm takes $O((|E|+|p E|) \cdot|V|)$ time. But, again, it can be shown that the number of pseudo-edges introduced is $O(|E|)$, thus, giving an $O(|E| \cdot|V|)$ time algorithm.
The proof of correctness involves two parts: In part 1, we show, by induction on the structure of the $U$ - and $\bar{U}$. Fragments, that when the algorithm claims the existence of certain disjoint paths, such paths, in fact, exist; In part 2 , we show by using U-Fragments as gadgets, that when the algorithm fails to muster enough evidence for the existence of certain disjoint paths, it is only when no such paths exist.

\section{REFERLNCES}

[1] A.V.Aho, J.E.HOPCROFT AND J.D.Ullman, The Design and Analysis of Computer Algorithms, Addison. Wesley, Reading, MA, 1974.

[2] D.BRAnd, "Detecting Sneak Paths in Transistor Networks", IBM Thomas J. Watson Research Center, Yorktown Heights, N. Y., 1983.

[3] S.Even, Graph Algorithms, Computer Science Press, Maryland 1979

[4] S.Even and R.E.TARJAn, "Network Flow and Testing Graph Connectivity", SIAM Journal of Computing, Vol. 4, No. 4, December 1975.

[5] E.FrANk, A Data Driven Multiprocessor for Switch Level Simulation of VLSI Circuits., Ph. D. Thesis (in preparation), 1984.

[6] J.HOPCROFT AND R.TARJAN, "Efficient Planarity Testing", Journal of the Association for Computing Machinery, Vol. 21, No. 4, October 1978.

[7] T.OHTsukI, "The Two Disjoint Path Problem and Wire Routing Design", in Graph Theory and Algorithms (Eds. N.Saito, T.Nishizeki), Springer 108, October 1980.

[8] Y. Perl and Y.ShIloACH, "Finding Two Disjoint Paths Between Two Pairs of Vertices in a Graph", Journal of the Association for Computing Machinery, Vol. 25, No. 1, January 1978.

[9] P.D.Seymour, "Disjoint Paths in Graphs", Discrete Mathematics, Vol. 29, No. 3, March 1980.

[10] Y.SHLLACH, "A Polynomial Solution to the Undirected Two Paths Problem", Journal of the Association for Computing Machinery, Vol. 27, No. 3, July 1980.

[11] R.TARJAN, "Depth-First Search and Linear Graph Algorithms", SIAM Journal of Computing, Vol. 1, No. 2, June 1972.

[12] R.E.TARJAN, Data Structures and Network Algorithms, Society for Industrial and Applied Mathematics, Philadclphia, Pa., 1983.

[13] W.T.TutTE, "Bridges and Hamiltonian circuits in planar graphs", Aequationes Mathematicae, 15, 1977.

[14] W.T.TUTTE, Graph Theory, Addison-Wesley Publishing Company, Menlo Park, California, 1984. 